$(85.7 \%)$, did not report oral sex $(85.7 \%)$, and were younger (median -24 years, IQR $=21-28 ; \mathrm{p}=0.013$ ) than pNG-negative participants (median -29 years, $\mathrm{IQR}=27-32$ ). The paired $(n=4)$ urogenital-pharyngeal isolates displayed the same antimicrobial susceptibility profile: ciprofloxacin-, tetracycline-, and penicillin-resistant and ceftriaxone- and cefixime-susceptible. None of the pharyngeal samples were positive for Chlamydia trachomatis and one sample was positive for Mycoplasma genitalium.

Conclusions To our knowledge, this is the first report of pNG in Ugandan men. NAATs were more sensitive than cultures for detection of pNG. In this pilot study, there was no difference in the susceptibility profiles of paired pharyngeal and urogenital NG isolates. Differences by age might be explained by differences in sexual behaviors or mucosal immunity. Additional studies are warranted to better define the epidemiology and microbiology of pNG infections in RLS.

\section{P151 SYSTEMATIC LITERATURE REVIEW AND QUANTITATIVE ANALYSIS OF HEALTH PROBLEMS ASSOCIATED WITH SEXUALLY TRANSMITTED NEISSERIA GONORRHOEAE INFECTION}

1J Whelan*, ${ }^{2} \mathrm{~J}$ Eeuwijk, ${ }^{2}$ E Bunge, ${ }^{3}$ E Beck. ${ }^{1}$ GSK Vaccines, Amsterdam, The Netherlands; ${ }^{2}$ Pallas health research and consultancy, Rotterdam, The Netherlands; ${ }^{3}$ GSK Vaccines, Wavre, Belgium

\subsection{6/sextrans-2021-sti.259}

Background Neisseria gonorrhoeae $(\mathrm{Ng})$ is the second most common sexually transmitted bacterial infection (STI), leading to serious health problems in men, women and newborns. While early antibiotic treatment is effective, infections are increasingly antibiotic-resistant. No systematic reviews present health problems associated with $\mathrm{Ng}$ infections or their likelihood of occurrence. The objective, therefore, was to conduct a systematic literature review to address these gaps.

Methods A systematic literature review was conducted of all studies with an English abstract published since 1950 (Pubmed)/1966 (Embase). The search included patients with a history of/current sexually transmitted $\mathrm{Ng}$ infection. Expected outcomes were defined from published reviews of gonorrhoea health problems. Observational studies with a control group were included. A decision tree determined the best quality studies for each outcome, prioritising generalisable populations, lab-confirmed diagnosis, clearly defined outcomes, no STI coinfections, adjusted analyses, and risk estimates. Where feasible, a meta-analysis was performed, otherwise the best quality study estimates were identified.

Results In total, 46 studies were included, and 22 health problems were identified. Of these problems, $\mathrm{Ng}$ infection was statistically significantly associated with preterm premature ruptures of membranes, preterm birth, low birth weight, stillbirth, infant death, neonatal ophthalmia, schizophrenia in offspring, pelvic inflammatory disease and subsequent tubal infertility, human immunodeficiency virus, and prostate cancer/ problems. High-quality evidence was generally lacking, with high heterogeneity across studies, and limited or inconclusive data on other health problems.

Conclusion $\mathrm{Ng}$ infection is associated with severe health problems in women, men and newborns. More high-quality comparative studies are needed to address the limitations in current knowledge.

\section{P152 COMMUNITY-BASED HIV TESTING IN THE NETHERLANDS: EXPERIENCES OF LAY PROVIDERS AND END USERS AT A RAPID HIV TEST CHECKPOINT}

E Op de Coul, R Spijker, A Żakowicz, M de Moraes, T Heijman, E Op de Coul*. Rivm, Bilthoven, The Netherlands

\subsection{6/sextrans-2021-sti.260}

Background The AIDS Healthcare Foundation (AHF-Checkpoint) in the Netherlands provides free 'walk-in', rapid HIV testing for key populations at onsite and pop-up locations. We explored the perspectives, experiences, and needs of lay providers and end users of this HIV test service, taking into account the WHO 5Cs (consent, confidentiality, counselling, correct results, connection-to-care) performance criteria for HIV test services.

Methods A qualitative evaluation with 15 semi-structured indepth interviews (April-June 2020) by telephone or video calling with ten lay providers and five end users from AHFCheckpoint. Recorded interviews were transcribed verbatim and thematically analysed.

Results Data analysis identified four domains: 1) accessibility of HIV testing, 2) quality of test procedures, 3) bridging (transitional care), and 4) future strategies for service delivery. AHF-Checkpoint fills a gap for key populations, including LGBTQ and refugees, who experience barriers to HIV testing at sexual health centres or GPs by providing anonymous, rapid testing. The level of trust between lay providers and end users was highly valued by end users. They also appreciated the low threshold to test, no waiting lists, no test costs or triaging that could include referral to another test location. Needs expressed by lay providers included more preparedness for emotionally charged situations and extra training to improve knowledge on STIs. Some end users expressed a need for a full STI test package at AHF-Checkpoint. Of the $5 \mathrm{Cs}$, consent, counselling, and correct results were realised, but confidentiality was sometimes difficult to achieve at popup locations, and referral barriers for confirmation testing (connection-to-care) were occasionally experienced by lay providers during weekends.

Conclusion AHF-Checkpoint was described as a convenient and easily accessible service by end users and lay providers. Of the WHO 5Cs, connection-to-care could be optimised to ensure HIV confirmation -and STI testing through a liaison approach with professionals from the regular healthcare sector.

\section{P155 CLINICAL PRESENTATION OF INCIDENT SYPHILIS AMONG MEN WHO HAVE SEX WITH MEN TAKING HIV PRE-EXPOSURE PROPHYLAXIS IN MELBOURNE, AUSTRALIA}

${ }^{1} \mathrm{~J}$ Peel* ${ }^{*},{ }^{1,2,3}{ }^{3}$ E Chow, ${ }^{1}$ I Denham, ${ }^{1} T$ Schmidt, ${ }^{1} \mathrm{~A}$ Buchanan, ${ }^{1,2} \mathrm{C}$ Fairley, ${ }^{4,5,6} \mathrm{D}$ Williamson, ${ }^{1} \mathrm{M}$ Bissessor, ${ }^{1,2} \mathrm{M}$ Chen. ${ }^{1}$ Melbourne Sexual Health Centre, Melbourne, Australia; ${ }^{2}$ Central Clinical School, Monash University, Melbourne, Australia; ${ }^{3}$ Centre for Epidemiology and Biostatistics, Melbourne School of Population and Global Health, The University of Melbourne, Melbourne, Australia; ${ }^{4}$ Microbiological Diagnostic Unit Public Health Laboratory, Department of Microbiology and Immunology, The University of Melbourne at The Peter Doherty Institute for Infection and Immunity, Melbourne, Australia; ${ }^{5}$ Department of Microbiology and Immunology, The University of Melbourne at The Peter Doherty Institute for Infection and Immunity, Melbourne, Australia; ${ }^{6}$ Department of Microbiology, Royal Melbourne Hospital, Melbourne, Australia

10.1136/sextrans-2021-sti.261 